International Journal of Pure and Applied Mathematics

Volume 88 No. 3 2013, 407-412

ISSN: 1311-8080 (printed version); ISSN: 1314-3395 (on-line version)

url: http://www.ijpam.eu

doi: http://dx.doi.org/10.12732/ijpam.v88i3.8

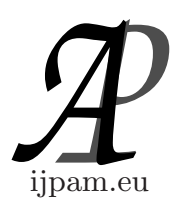

\title{
ON POINT SPECTRUM OF SUBSPACE-HYPERCYCLIC OPERATORS
}

\author{
Mansooreh Moosapoor \\ Farhangian University \\ Postcode 4166616711, Rasht, IRAN
}

\begin{abstract}
It is well known that if $T$ is a hypercyclic operator, then $\sigma_{p}\left(T^{*}\right)=$ $\phi$. We prove in this paper that this is not true for subspace-hypercyclic operators. We show that if $T$ is subspace-hypercyclic, then $\sigma_{p}\left(T^{*}\right)$ may be empty or not. Moreover we show that for every scalar $\lambda$ with $|\lambda|>1$, there exists a subspace-hypercyclic operator $T$ such that $\|T\|=|\lambda|$ and $\sigma_{p}\left(T^{*}\right) \neq \phi$.
\end{abstract}

AMS Subject Classification: 47A16, 47B37, 37B99

Key Words: subspace-hypercyclic operators, point spectrum

\section{Introduction and Preliminaries}

Let $X$ be a Banach space. An operator $T$ on $X$ is hypercyclic if there exists a vector $x \in X$ whose orbit under $T$, orb $(T, x)=\left\{x, T x, T^{2} x, \ldots\right\}$, is dense in $X$. Such a vector $x$ is called a hypercyclic vector for $T$.

By Ansari's theorem in [1], hypercyclic operators exist on every separable and infinite dimensional Frechet space. Hypercyclic operators have been actively studied for more than twenty years. One can refer to [2-5] for more information.

Recently, B. F. Madore and R. A. Martnez-Avendano in [8] introduced the concept of subspace-hypercyclicity for an operator. We recall some preliminaries from [8] in this paper.

Received: July 10, 2013

(c) 2013 Academic Publications, Ltd. url: www.acadpubl.eu 
In all of paper $H$ always denotes a separable Hilbert space over $C$, the field of complex numbers and $M$ is always a non-zero topologically closed subspace of $H . B(H)$ is the space of bounded linear operators acting on $H$ and we say its elements, operators.

Let us recall some definitions and theorems from [8].

Definition 1.1. Let $T \in B(H)$ and $M$ be a closed subspace of $H$. We say $T$ is $M$-hypercyclic, if there exists $x \in H$ such that $\operatorname{orb}(T, x) \cap M$ is dense in $M$. Such a vector $x$ is called a $M$-hypercyclic vector for $T$.

Definition 1.2. Let $T \in B(H)$ and $M$ be a closed subspace of $H$. We say $T$ is $M$-transitive, if for any non-empty open sets $U, V \subseteq M$, both relatively open, there exists $n \in N_{0}$ such that $U \cap T^{-n}(V)$ contains a relatively open non-empty subset of $M$.

Theorem 1.3. Let $T \in B(H)$. The following conditions are equivalent:

(i) $T$ is subspace-transitive with respect to $M$.

(ii) for any non-empty sets $U \subseteq M, V \subseteq M$ both relatively open, there exists $n \in N_{0}$ such that $T^{-n}(U) \cap V$ is a relatively open non-empty subset of $M$.

(iii) for any non-empty sets $U \subseteq M, V \subseteq M$ both relatively open, there exists $n \in N_{0}$ such that $T^{-n}(U) \cap V$ is non-empty and $T^{n}(M) \subseteq M$.

Lemma 1.4. Let $T \in B(H)$ and $M$ be a closed subspace of $H$. If $T$ is $M$-transitive, then $H C(T, M)=\left(\bigcap_{j=1}^{\infty} \bigcup_{n=1}^{\infty} T^{-n}\left(B_{j}\right)\right)$ is a dense subset of $M$, where $H C(T, M)$ is the set of $M$-hypercyclic vectors for $T$ and $\left\{B_{j}\right\}$ is a countable open basis for the relative topology of $M$ as a subspace of $H$.

An immediate corollary of Lemma 1.4 is that subspace-transitive operators are subspace-hypercyclic.

Theorem 1.5. Let $T \in B(H)$ and $M$ be a nonzero subspace of $H$. If $T$ is is subspace-hypercyclic for $M$, then $T$ is subspace-hypercyclic for $M$.

The following theorem shows that subspace-hypercyclicity, like hypercyclicity, is a purely infinite dimensional concept.

Theorem 1.6. Let $H$ be finite-dimensional. If $T \in B(H)$, then $T$ is not subspace-hypercyclic for any $M$.

Theorem 1.7. Let $T \in B(H)$. If $T$ is subspace-hypercyclic for $M$, then $M$ is not finite dimensional. 
Theorem 1.8. Let $T \in B(H)$. If $T$ is subspace-hypercyclic for some subspace, then $\sigma(T) \cap S^{1} \neq \phi$.

By Theorem 1.8, if an operator is subspace-hypercyclic, then its spectral radius must be greater or equal to 1 .

\section{Main Results}

If $T \in B(H)$ is a hypercyclic operator, then the point spectrum of $T^{*}$, is empty by [6]. That means we have:

$$
\sigma_{p}\left(T^{*}\right)=\{\lambda \in C: T-\lambda I \text { is not one to one }\}=\phi .
$$

However this is not true for subspace-hypercyclic operators as the following example shows.

Example 2.1. Let $A \in B(H)$ be a hypercyclic operator and $x$ be a hypercyclic vector for $A$ and let $T=A \oplus \lambda_{1} I \oplus \lambda_{2} I \oplus \ldots \oplus \lambda_{n} I$, where $\lambda_{1}, \lambda_{2}, \ldots, \lambda_{n}$ are complex numbers and $I$ is the identity operator on $H$. Then it is clear that $T$ is a subspace-hypercyclic operator with respect to $M:=H \oplus\{0\} \oplus \ldots \oplus\{0\}$ and $x \oplus\{0\} \oplus \ldots \oplus\{0\}$ is a $M$-hypercyclic vector for it. Also it is clear that $\left\{\bar{\lambda}_{1}, \bar{\lambda}_{2}, \ldots, \bar{\lambda}_{n}\right\} \subseteq \sigma_{p}\left(T^{*}\right)$.

Furthermore for every bounded sequence of scalars, we can construct an operator $T$ such that $\sigma_{p}\left(T^{*}\right)$ contains that sequence.

Corollary 2.2. For every bounded sequence $\left\{\lambda_{n}\right\}$ of scalars, we can find a subspace-hypercyclic operator $T$ such that $\left\{\lambda_{n}\right\} \subseteq \sigma_{p}\left(T^{*}\right)$.

Proof. It is sufficient to consider $T=A \oplus \bar{\lambda}_{1} I \oplus \bar{\lambda}_{2} I \oplus \ldots \oplus \bar{\lambda}_{n} I \oplus \ldots$, where $A \in B(H)$ is hypercyclic.

Remark 2.3. In [8], it is proved that if $T \in B(H)$ is subspace-hypercyclic for $M$, then $\operatorname{ker}\left(T^{*}-\lambda\right)^{p} \subseteq M^{\perp}$ for all $\lambda \in C$ and all $p \in N$. By Example 2.1, we can say that in some cases $\operatorname{ker}\left(T^{*}-\lambda\right)^{p}$ is also non-empty.

Let $B$ be the backward shift on $l^{2}$. So for every $\left(x_{0}, x_{1}, x_{2}, \ldots\right) \in l^{2}$,

$$
B\left(x_{0}, x_{1}, x_{2}, \ldots\right)=\left(x_{1}, x_{2}, \ldots\right) .
$$

Rolweicz showed in [10] that for every scalar $\lambda$ with $|\lambda|>1, \lambda B$ is hypercyclic. We use this fact in the proof of next theorem.

Theorem 2.4. For every scalar $\lambda \in C$ with $|\lambda|>1$, there exists a subspace-hypercyclic operator $T$ such that $\|T\|=|\lambda|$ and $\sigma_{p}\left(T^{*}\right) \neq \phi$. 
Proof. Let $A=\lambda B$, where $B$ is the backward shift on $l^{2}$ and $|\lambda|>1$. Let $\lambda_{1}$ be a complex number such that $\left|\lambda_{1}\right| \leq|\lambda|$. Then $T=A \oplus \lambda_{1} I \in B\left(l^{2} \oplus l^{2}\right)$ is a subspace-hypercyclic operator with respect to $M=l^{2} \oplus\{0\}$. Moreover

$$
\|T\|=\max \left\{|\lambda|,\left|\lambda_{1}\right|\right\}=|\lambda| .
$$

Like Example 2.1, $\sigma_{p}\left(T^{*}\right) \neq \phi$ and similar to Example 2.2 in [8], $T$ is not hypercyclic.

Clearly $T$ is not hypercyclic, since $\sigma_{p}\left(T^{*}\right) \neq \phi$.

Theorem 2.5. For every scalar $\alpha$ with $|\alpha|>1$, there exists a subspacehypercyclic operator $T$ such that, $T$ is not hypercyclic, $\rho(T)=|\alpha|$ and $\sigma_{p}\left(T^{*}\right) \neq$ $\phi$.

Proof. Let $T=\alpha B \oplus \lambda I$ where $B$ is the backward shift on $l^{2}$ and $1<|\lambda|<$ $|\alpha|$. As we know $T$ is subspace- hypercyclic operator but not hypercyclic. Also we have:

$$
\begin{gathered}
\rho(\alpha B \oplus \lambda I)=\lim _{n \rightarrow \infty}\left\|(\alpha B \oplus \lambda I)^{n}\right\|^{\frac{1}{n}}=\lim _{n \rightarrow \infty}\left\|\left(\alpha^{n} B^{n} \oplus \lambda^{n} I^{n}\right)\right\|^{\frac{1}{n}} \\
=\lim _{n \rightarrow \infty}\left(\max \left\{\left(\left|\alpha^{n}\right|,\left|\lambda^{n}\right|\right)\right\}\right)^{\frac{1}{n}}=\lim _{n \rightarrow \infty}\left(|\alpha|^{n}\right)^{\frac{1}{n}}=|\alpha| .
\end{gathered}
$$

Lemma 2.6. (see [9]) Let $T \in B(H)$ and $\lambda_{1}, \lambda_{2}, \ldots, \lambda_{n}$ be distict non-zero eigenvalues of $T$, and let $x_{1}, x_{2}, \ldots, x_{n}$ be corresponding non-zero eigenvectors. Then the vectors $x_{i}(i \in N)$ are linearly independent.

Theorem 2.7. Let $T$ be a subspace-hypercyclic operator of the form $T=A \oplus \lambda_{1} I \oplus \lambda_{2} I \oplus \ldots \oplus \lambda_{n-1} I \in B(H \oplus H \oplus \ldots \oplus H)$, where $A \in B(H)$ is hypercyclic, $\lambda_{i}$ 's $(1 \leq i \leq n-1)$ are scalars and $I$ is the identity operator on $H$. Then $T^{*}$ has an invariant and non-trivial subspace of $H \oplus H \oplus \ldots \oplus H$.

Proof. First we note that $\left\{\bar{\lambda}_{1}, \ldots, \bar{\lambda}_{n-1}\right\} \subseteq \sigma_{p}\left(T^{*}\right)$. Let $x_{i}$ be corresponding non-zero eigenvector for $\bar{\lambda}_{i}$ where $1 \leq i \leq n-1$.

If we consider $M:=\operatorname{span}\left\{x_{1}, x_{2}, \ldots, x_{n-1}\right\}$, then $M$ is closed and invariant under $T^{*}$. Clearly $M \neq H \oplus H \oplus \ldots \oplus H$, since $M$ is finite dimensional.

Remark 2.8. Note that if we replace all $\lambda_{i} I^{\prime}$ 's $(1 \leq i \leq n-2)$ with hypercyclic operators on $H$, the theorem remains true.

In the following example, we show that the point spectrum of the adjoint of a subspace-hypercyclic operator may be empty. 
Example 2.9. Let $A, B \in B(H)$ be hypercyclic operators. Then $T=$ $A \oplus B$ is a subspace-hypercyclic operator with respect to $M:=H \oplus\{0\}$.

Let $\lambda \in \sigma_{p}\left(T^{*}\right)$ and $x \oplus y$ be an eigenvector correspondence to $\lambda$ and without lose of generality we can assume that $x$ and $y$ are non-zero. So

$$
T^{*}(x \oplus y)=\left(A^{*} x \oplus B^{*} y\right)=\lambda(x \oplus y) .
$$

Hence $A^{*} x=\lambda x$ and $B^{*} y=\lambda y$. That means $\sigma_{p}\left(A^{*}\right) \neq \phi$ and $\sigma_{p}\left(B^{*}\right) \neq \phi$. But this is a contradiction. So $\sigma_{p}\left(T^{*}\right)$ must be empty.

From the above example, one can obtain subspace-hypercyclic operators with empty point spectrum.

For example let $\lambda_{1}, \lambda_{2}$ be two scalars such that $\left|\lambda_{1}\right|>1$ and $\left|\lambda_{2}\right|>1$, and let $B$ be the backward shift on $l^{2}$. Then if we consider $T=\lambda_{1} B \oplus \lambda_{2} B$, then it is clear that $T$ is subspace-hypercyclic with respect to $M:=l^{2} \oplus\{0\}$. Note that the point spectrum of $T^{*}$ is empty by Example 2.9, since $\lambda_{1} B$ and $\lambda_{2} B$ are hypercyclic operators on $l^{2}$.

Now we can say the following lemma:

Lemma 2.10. There is a subspace-hypercyclic operator $T$ such that, $\sigma_{p}\left(T^{*}\right)$ is empty.

In fact we can find many of these operators by Example 2.9.

Theorem 2.11. If $T \in B(H)$ is a subspace-hypercyclic operator, then $\sigma_{p}\left(T^{*}\right)$ may be empty or not.

Proof. The proof is clear by Theorem 2.4 and Lemma 2.10 .

By Theorem 1.5, subspace-transitive operators are subspace-hypercyclic. So we have the following corollary:

Corollary 2.12. If $T \in B(H)$ is a subspace-transitive operator, then $\sigma_{p}\left(T^{*}\right)$ may be empty or not.

\section{References}

[1] Shamim I. Ansari, Existense of hypercyclic operators on topological vector spaces, J. Func. Anal., 148 (1997), 384-390, doi: 10.1006/jfan.1996.3093.

[2] F. Bayart, E. Matheron, Dynamics of linear operators, Cambridge University Press (2009). 
[3] G. Godefroy, J. H. Shapiro, Operators with dense, invariant, cyclic vector manifolds, J. Funct. Anal., 98 (1991), 229-269, doi: 10.1016/00221236(91)90078-J.

[4] K.-G. Grosse-Erdmann, A.Peris Manguillot, Linear chaos, Springer (2011).

[5] K.-G. Grosse-Erdmann, Universal families and hypercyclic operators, Bull. Amer. Math. Soc. (N.S.), 36 (1999), 345-381.

[6] Domingo A. Herrero, Limits of hypercyclic and supercyclic operators, $J$. Func. Anal., 99 (1991), 179-190, doi: 10.1016/0022-1236(91)90058-D.

[7] C.M. Le, On subspace-hypercyclic operators, Proc. Amer. Math.Soc., B9 (2011), 2847-2852, doi: 10.1090/ S0002-9939-2011-10754-8.

[8] B.F. Madore, R.A. Martínez-Avendaño, Subspace hypercyclicity, J. Math. Anal. Appl., 373 (2011), 502-511, doi: 10.1016/j.jmaa.2010.07.049.

[9] V. Muller, Spectral theory of linear operators, Birkhauser Verlag AG (2007).

[10] S. Rolewicz, On orbits of elements, Studia Math., 33 (1969), 17-22. 